\title{
HEDEN'S BOUND ON THE TAIL OF A VECTOR SPACE PARTITION
}

\author{
SASCHA KURZ
}

\begin{abstract}
A vector space partition of $\mathbb{F}_{q}^{v}$ is a collection of subspaces such that every non-zero vector is contained in a unique element. We improve a lower bound of Heden on the number of elements of the smallest occurring dimension.
\end{abstract}

\section{INTRODUCTION}

In this note we translate Heden [5] into geometry and find that the same theory now only takes a small fraction of the space. Having decoded the approach using mixed perfect 1-codes, we go along the lines of [7] and improve Heden's result a little. In [7] analytic solutions of linear programming methods for projective linear codes or sets of points have been applied in order to compute upper bounds for partial spreads. Interestingly enough, the very same happened in [3], where the authors translated and improved a lower bound of Heden on the size of maximal partial line spreads.

Let $q>1$ be a prime power and $v$ a positive integer. A vector space partition $\mathcal{P}$ of $\mathbb{F}_{q}^{v}$ is a collection of subspaces with the property that every non-zero vector is contained in a unique member of $\mathcal{P}$. If $\mathcal{P}$ contains $m_{d}$ subspaces of dimension $d$, then $\mathcal{P}$ is of type $k^{m_{k}} \ldots 1^{m_{1}}$. We may leave out some of the cases with $m_{d}=0$. If $d_{1}$ is the smallest dimension with $m_{d_{1}} \neq 0$, we call $m_{d_{1}}$ the length of the tail. Subspaces of dimension $d$ are also called $d$-spaces. 1 -spaces are called points and each $k$-space contains $\left[\begin{array}{l}k \\ 1\end{array}\right]_{q}:=\frac{q^{k}-1}{q-1}$ points. Heden's main result is:

Theorem 1. (Theorem 1 in [5]) Let $\mathcal{P}$ be a vector space partition of type $d_{l}{ }^{u_{l}} \ldots d_{2}{ }^{u_{2}} d_{1}{ }^{u_{1}}$ of $\mathbb{F}_{q}^{v}$, where $u_{1}, u_{2}>0$.

(i) If $q^{d_{2}-d_{1}}$ does not divide $u_{1}$ and if $d_{2}<2 d_{1}$, then $u_{1} \geq q^{d_{1}}+1$;

(ii) if $q^{d_{2}-d_{1}}$ does not divide $u_{1}$ and if $d_{2} \geq 2 d_{1}$, then either $d_{1}$ divides $d_{2}$ and $u_{1}=\left[\begin{array}{c}d_{2} \\ 1\end{array}\right]_{q} /\left[\begin{array}{c}d_{1} \\ 1\end{array}\right]_{q}$ or $u_{1}>2 q^{d_{2}-d_{1}}$;

(iii) if $q^{d_{2}-d_{1}}$ divides $u_{1}$ and $d_{2}<2 d_{1}$, then $u_{1} \geq q^{d_{2}}-q^{d_{1}}+q^{d_{2}-d_{1}}$;

(iv) if $q^{d_{2}-d_{1}}$ divides $u_{1}$ and $d_{2} \geq 2 d_{1}$, then $u_{1} \geq q^{d_{2}}$.

The other theorems of [5] are as follows: Theorems 2 and 3 classify the possible sets of $d_{1}$-spaces for $u_{1}=q^{d_{1}}+1$ and $u_{1}=\left[\begin{array}{c}d_{2} \\ 1\end{array}\right]_{q} /\left[\begin{array}{c}d_{1} \\ 1\end{array}\right]_{q}$, respectively. Theorem 4 is the direct application of Theorem 3 and Theorem 1(i).

\section{SETS OF DISJOINT $k$-SPACES AND THEIR INCIDENCES WITH HYPERPLANES}

For a positive integer $k$ let $\mathcal{N}$ be a set of pairwise disjoint $k$-spaces in $\mathbb{F}_{q}^{v}$, where $v$ is minimal. By $a_{i}$ we denote the number of hyperplanes $H$ of $\mathbb{F}_{q}^{v}$ with $\#(\mathcal{N} \cap H):=\#\{U \in \mathcal{N}: U \leq H\}=i$ and set $n:=\# \mathcal{N}$. Double-counting the incidences of the tuples $(H),\left(B_{1}, H\right)$, and $\left(B_{1}, B_{2}, H\right)$, where $H$ is a hyperplane and $B_{1} \neq B_{2}$ are elements of $\mathcal{N}$ contained in $H$ gives:

$$
\sum_{i=0}^{n-1} a_{i}=\left[\begin{array}{l}
v \\
1
\end{array}\right]_{q}, \quad \sum_{i=0}^{n-1} i a_{i}=n \cdot\left[\begin{array}{c}
v-k \\
1
\end{array}\right]_{q}, \quad \text { and } \quad \sum_{i=0}^{n-1} i(i-1) a_{i}=n(n-1) \cdot\left[\begin{array}{c}
v-2 k \\
1
\end{array}\right]_{q} .
$$

For three different elements $B_{1}, B_{2}, B_{3}$ of $\mathcal{N}$ their $\operatorname{span}\left\langle B_{1}, B_{2}, B_{3}\right\rangle$ has a dimension $i$ between $2 k$ and $3 k$. Denoting the number of corresponding triples by $b_{i}$, double-counting gives:

$$
\sum_{i=0}^{n-1} i(i-1)(i-2) a_{i}=\sum_{i=2 k}^{3 k} b_{i}\left[\begin{array}{c}
v-i \\
1
\end{array}\right]_{q} \text { and } \sum_{i=2 k}^{3 k} b_{i}=n(n-1)(n-2) .
$$

Given parameters $q, k, n$, and $v$ the so-called (integer) linear programming method, developed for association schemes by Delsarte [4], asks for a solution of the equation system given by (1) and (2) with $a_{i}, b_{i} \in \mathbb{R}_{\geq 0}\left(a_{i}, b_{i} \in \mathbb{N}\right)$. If no solution exists, then no corresponding set $\mathcal{N}$ can exist. For $k=1$ one can take the elements of $\overline{\mathcal{N}}$ as the columns of a generator matrix of a projective linear code over $\mathbb{F}_{q}$. In this case, the equations from (1) and (2) correspond to the first four MacWilliams identities, see e.g. [6].

Lemma 2. If $a_{i}=0$ for all $i \neq r>0$ and $k<v$ in the above setting, then there exists an integer $s \geq 2$ with $v=$ sk and $\mathcal{N}$ consists of $\frac{q^{v}-1}{q^{k}-1}$ disjoint $k$-spaces partitioning $\mathbb{F}_{q}^{v}$. Additionally we have $r=\frac{q^{v-k}-1}{q^{k}-1}$.

* Grant KU 2430/3-1 - Integer Linear Programming Models for Subspace Codes and Finite Geometry - German Research Foundation. 
Proof. Solving $\left\{1\right.$ for $r, a_{r}$, and $n$ gives $n=\frac{q^{2 v-k}-q^{v}-q^{v-k}+1}{q^{v}-q^{v-k}-q^{k}+1}$. Writing $v=s k+t$ with $s, t \in \mathbb{N}$ and $0 \leq t<k$ we obtain $n=\sum_{i=1}^{s} q^{v-i k}+\frac{q^{v-k+t}-q^{v-k}-q^{t}+1}{q^{v}-q^{v-k}-q^{k}+1}$. Since $n \in \mathbb{N}$ and $0 \leq q^{v-k+t}-q^{v-k}-q^{t}+1<q^{v}-q^{v-k}-q^{k}+1$ we have $q^{v-k+t}-q^{v-k}-q^{t}+1=0$ so that $t=0$ and $n=\frac{q^{v}-1}{q^{k}-1}$. Counting points gives that $\mathcal{N}$ partitions $\mathbb{F}_{q}^{v}$.

We remark that $r=0$ forces $n \in\{0,1\}$ so that $\mathcal{N}$ is empty or consists of a single $k$-space in $\mathbb{F}_{q}^{k}$ and $v=k$ implies the latter case. So, this degenerated cases correspond to $s \in\{0,1\}$ in Lemma 2] As pointed out after [5, Theorem 2], such results can be proved in different ways. While the case that only one $a_{i}$ is non-zero is rather special, we can show that many $a_{i}$ are equal to zero in our setting.

Lemma 3. Let $\mathcal{P}$ be a vector space partition of type $d_{l}{ }^{u_{1}} \ldots d_{2}{ }^{u_{2}} d_{1}{ }^{u_{1}}$ of $\mathbb{F}_{q}^{v}$, where $u_{1}, u_{2}>0$, and $\mathcal{N}$ be the set of $d_{1}$-spaces. Then, we have $\# \mathcal{N} \equiv \#(\mathcal{N} \cap H)\left(\bmod q^{d_{2}-d_{1}}\right)$ for every hyperplane $H$ of $\mathbb{F}_{q}^{v}$.

Proof. For each $U \in \mathcal{P}$ we have $\operatorname{dim}(U \cap H) \in\{\operatorname{dim}(U), \operatorname{dim}(U)-1\}$. So counting points in $\mathbb{F}_{q}^{v}$ and $H$ gives the existence of integers $a, a^{\prime}$ with $m \cdot\left[\begin{array}{c}d_{2} \\ 1\end{array}\right]_{q}+a q^{d_{2}}+u_{1}\left[\begin{array}{c}d_{1} \\ 1\end{array}\right]_{q}=\left[\begin{array}{l}v \\ 1\end{array}\right]_{q}$ and $m \cdot\left[\begin{array}{c}d_{2}-1 \\ 1\end{array}\right]_{q}+a^{\prime} q^{d_{2}-1}+u_{1}^{\prime} q^{d_{1}-1}+u_{1}\left[\begin{array}{c}d_{1}-1 \\ 1\end{array}\right]_{q}=$ $\left[\begin{array}{c}v-1 \\ 1\end{array}\right]_{q}$, where $m:=\sum_{i=2}^{l} u_{i}$ and $u_{1}^{\prime}:=\#(\mathcal{N} \cap H)$. By subtraction we obtain $m q^{d_{2}-1}+a q^{d_{2}}-a^{\prime} q^{d_{2}-1}+u_{1} q^{d_{1}-1}-$ $u_{1}^{\prime} q^{d_{1}-1}=q^{v-1}$, so that $u_{1} q^{d_{1}-1} \equiv u_{1}^{\prime} q^{d_{1}-1}\left(\bmod q^{d_{2}-1}\right)$.

Definition 4. Let $\mathcal{N}$ be a set of $k$-spaces in $\mathbb{F}_{q}^{v}$. If there exists a positive integer $r$ such that $a_{i}$ is non-zero only if $\# \mathcal{N}-i$ is divisible by $q^{r}$ and the $k$-spaces are pairwise disjoint, then we call $\mathcal{N} q^{r}$-divisible.

Using the notation of Lemma $3, \mathcal{N}$ is $q^{d_{2}-d_{1}}$-divisible. For $d_{1}=1$, taking the elements of $\mathcal{N}$ as columns of a generator matrix, we obtain a projective linear code, whose Hamming weights are divisible by $q^{d_{2}-1}$.

Lemma 5. For a $q^{r}$-divisible set $\mathcal{N}$ of $k$-spaces in $\mathbb{F}_{q}^{v}$, there exists a hyperplane $H$ with $\#(\mathcal{N} \cap H) \leq n / q^{k}$.

PROOF. Let $i$ be the smallest index with $a_{i} \neq 0$. Then, the first two equations of $[1]$ are equivalent to $\sum_{j \geq 0} a_{i+q^{r} j}=$ $\left[\begin{array}{l}v \\ 1\end{array}\right]_{q}$ and $\sum_{j \geq 0}\left(i+q^{r} j\right) \cdot a_{i+q^{r} j}=n\left[\begin{array}{c}v-k \\ 1\end{array}\right]_{q}$. Subtracting $i$ times the first equation from the second equation gives $\sum_{j>0} q^{r} j a_{i+q^{r} j}=n \cdot \frac{q^{v-k}-1}{q-1}-i \cdot \frac{q^{v}-1}{q-1}$. Since the left-hand side is non-negative, we have $i \leq \frac{q^{v-k}-1}{q^{v}-1} \cdot n \leq \frac{n}{q^{k}}$.

Stated less technical, the proof of Lemma 5 is given by the fact that the hyperplane with the minimum number of $k$-spaces contains at most as many $k$-spaces as the average number of $k$-spaces per hyperplane.

Lemma 6. Let $m \in \mathbb{Z}$ and $\mathcal{N}$ be a $q^{r}$-divisible set of $k$-spaces in $\mathbb{F}_{q}^{v}$. Then, $\tau\left(n, q^{r}, q^{k}, m\right) \cdot q^{v-2 k-2 r}-m(m-1) \geq 0$, where $\tau(n, \Delta, u, m):=\Delta^{2} u^{2} m(m-1)-n(2 m-1) u(u-1) \Delta+n(u-1)(n(u-1)+1)$.

PROOF. With $y=q^{v-2 k}, u=q^{k}$, and $\Delta=q^{r}$, we can rewrite the equations of $[1]$ to $u^{2} y-1=(q-1) \sum_{i \in \mathbb{Z}} a_{i}$, $n \cdot(u y-1)=(q-1) \sum_{i \in \mathbb{Z}} i a_{i}$, and $n(n-1) \cdot(y-1)=\sum_{i \in \mathbb{Z}} i(i-1) a_{i} \cdot(n-m \Delta)(n-(m-1) \Delta)$ times the first minus $2 n-(2 m-1) \Delta-1$ times the second plus the third equation gives $y \cdot \tau(n, \Delta, u, m)-\Delta^{2} m(m-1)=$ $(q-1) \sum_{i \in \mathbb{Z}}(n-m \Delta-i)(n-(m-1) \Delta-i) a_{i}=(q-1) \sum_{h \in \mathbb{Z}} \Delta^{2}(m-h)(m-h+1) a_{n-h \Delta} \geq 0$.

Lemma 7. If $\mathcal{N}$ is a q-divisible set of $k$-spaces in $\mathbb{F}_{q}^{v}$ of cardinality $q^{k}+1$, then $\mathcal{N}$ partitions $\mathbb{F}_{q}^{2 k}$.

Proof. Setting $c_{i}:=(q-1) a_{1+i q}$ and $l:=q^{k-1}-1$ we can rewrite the equations of 11 to $\sum_{i=0}^{l} c_{i}=q^{v}-1$, $\sum_{i=0}^{l}(1+i q) c_{i}=\left(q^{k}+1\right)\left(q^{v-k}-1\right)$, and $\sum_{i=0}^{l} i q(1+i q) c_{i}=\left(q^{k}+1\right) q^{k}\left(q^{v-2 k}-1\right)$. Since $q l+1$ times the second minus $q l+1$ times the first minus the third equation gives $0 \leq \sum_{i=0}^{l} i q^{2}(l-i) c_{i}=-q^{k+1}\left(q^{v-2 k}-1\right)$, we have $v=2 k$. Every point of $\mathbb{F}_{q}^{v}$ is covered by an element from $\mathcal{N}$ due to $\left[\begin{array}{c}2 k \\ 1\end{array}\right]_{q} /\left[\begin{array}{c}k \\ 1\end{array}\right]_{q}=q^{k}+1$.

\section{PROOF OF HEDEN'S RESUlTS AND FURTHER IMPROVEMENTS}

Let $\mathcal{P}$ be a vector space partition of type $d_{l}{ }^{u_{l}} \ldots d_{2}{ }^{u_{2}} d_{1}{ }^{u_{1}}$ of $\mathbb{F}_{q}^{v}$, where $u_{1}, u_{2}>0$, and $\mathcal{N}$ the set of $d_{1}$-spaces.

Assume that $q^{d_{2}-d_{1}}$ does not divide $u_{1}$. We have $\#(\mathcal{N} \cap H) \geq 1$ for every hyperplane $H$ due to Lemma 3 , so that Lemma 5 gives $u_{1} \geq q^{d_{1}}$. Thus, we have $u_{1} \geq q^{d_{1}}+1$ and can apply Lemma 7. If $u_{1}<2 q^{d_{2}-d_{1}}$ we can apply Lemma 2 so that either $d_{2}$ divides $d_{1}$ and $u_{1}=\left(q^{d_{2}}-1\right) /\left(q^{d_{1}}-1\right)$ or $u_{1}>2 q^{d_{2}-\overline{d_{1}}}$.

Assume that $q^{d_{2}-d_{1}}$ divides $u_{1}$. Setting $\Delta=q^{d_{2}-d_{1}}, u=q^{d_{1}}, n=l \Delta$, and $m=$ for some integer $l$, we conclude $\tau(n, \Delta, u, m)=l \Delta(\Delta l-\Delta u+u-1) \geq 0$ from Lemma 6, so that $l \geq\left\lceil u-\frac{u}{\Delta}+\frac{1}{\Delta}\right\rceil$. The right-hand side is equal to $u=q^{d_{1}}$ if $d_{2} \geq 2 d_{1}$ and to $u-u / \Delta+1=q^{d_{1}}-q^{2 d_{1}-d_{2}}+1$ otherwise, which is equivalent to $n \geq q^{d_{2}}$ and $n \geq q^{d_{2}}-q^{d_{1}}+q^{d_{2}-d_{1}}$. We remark that equality is achievable in the latter case via the 2 -weight codes constructed in [2] (with parameters $n^{\prime}=d_{1}$ and $m=d_{2}-d_{1}$ ). We do not know whether the corresponding $q^{d_{2}-d_{1}}$-divisible set of $d_{1}$-spaces can be realized as a vector space partition of $\left.\mathbb{F}_{q}^{v}\right|^{7}$ For the first case a construction is given by lifted maximum rank distance codes, cf. [5, Example 1].

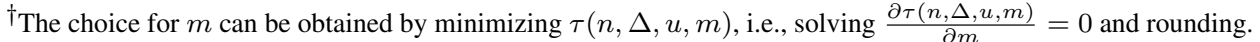

${ }^{\ddagger}$ A suitable test case might be to decide whether a vector space partition of type $4^{4} 3^{135} 2^{6}$ exists in $\mathbb{F}_{2}^{10}$.
} 
The above comprises [5, Theorems 1-4]. Just Theorem[1 (i), for the case where $d_{1}$ does not divide $d_{2}$, leaves some space for improving the lower bound on $u_{1}$. To that end we analyze Lemma 6 in more detail.

Proposition 8. Let $\mathcal{N}$ be a $q^{r}$-divisible set of $k$-spaces in $\mathbb{F}_{q}^{v}, u=q^{k}$ and $\Delta=q^{r}$. Then, $n \notin\left[1, \frac{q^{k+r}-1}{q^{r}-1}\right)$ and $n \notin$ $\left[\left\lceil\frac{1}{u-1} \cdot\left(\Delta u m-\frac{\Delta u+1}{2}-\frac{1}{2} \sqrt{\omega}\right)\right],\left\lfloor\frac{1}{u-1} \cdot\left(\Delta u m-\frac{\Delta u+1}{2}+\frac{1}{2} \sqrt{\omega}\right)\right\rfloor\right]$, where $\omega=(\Delta u-2 m)^{2}+\left(2 \Delta u+1-4 m^{2}\right)$, for all $m \in \mathbb{N}$ with $2 \leq m \leq\left\lfloor\frac{\Delta u}{4}+\frac{1}{2}+\frac{1}{4 \Delta u}\right\rfloor$.

Proof. We set $\bar{\Delta}=\Delta u$ and $\bar{n}=n(u-1)$ so that $\tau(n, \Delta, u, m)=\bar{\Delta}^{2} m(m-1)-\bar{n} \bar{\Delta}(2 m-1)+\bar{n}(\bar{n}+1)$. We have $\tau(n, \Delta, u, m) \leq 0$ iff $\left|\bar{n}-\bar{\Delta} m+\frac{\bar{\Delta}+1}{2}\right| \leq \frac{1}{2} \sqrt{\bar{\Delta}^{2}-4 m \bar{\Delta}+2 \bar{\Delta}+1}$ and $m \leq \frac{\bar{\Delta}}{4}+\frac{1}{2}+\frac{1}{4 \bar{\Delta}}$. Rewriting and applying Lemma 6 with $1 \leq m \leq\left\lfloor\frac{\Delta u}{4}+\frac{1}{2}+\frac{1}{4 \Delta u}\right\rfloor$ gives the result since $m(m-1)>0$ for $m \geq 2$.

Proposition 9. Let $\mathcal{N}$ be a $q^{r}$-divisible set of $k$-spaces in $\mathbb{F}_{q}^{v}$, where $r=a k+b$ with $a, b \in \mathbb{N}, 0<b<k$ and $a \geq 1$. Then, $n \geq \frac{q^{(a+2) k}-1}{q^{k}-1}=q^{r} \cdot q^{k-b}+\frac{q^{r} \cdot q^{k-b}-1}{q^{k}-1}=\Delta q^{k-b}+q^{k} \Theta+1$, where $\Delta:=q^{r}$ and $\Theta:=\frac{q^{a k}-1}{q^{k}-1}$.

Proof. From Lemma 2 we conclude $n \geq 2 q^{r}$ and set $u=q^{k}$. For $2 \leq m \leq q^{k-b}$ we have $2 \Delta u+1-4 m^{2}>0$, so that Proposition 8 gives $n \notin\left[\left\lceil\frac{\Delta u(m-1)-1 / 2+m}{u-1}\right\rceil,\left\lfloor\frac{\Delta u m-1 / 2-m}{u-1}\right\rfloor\right]$. Since $\Delta(m-1) \leq\left\lceil\frac{\Delta u(m-1)-1 / 2+m}{u-1}\right\rceil=$ $\Delta(m-1)+\left\lceil\frac{\Delta(m-1)-1 / 2+m}{u-1}\right\rceil \leq \Delta m$ and $\left\lfloor\frac{\Delta u m-1 / 2-m}{u-1}\right\rfloor=\Delta m+m q^{b} \Theta+\left\lfloor\frac{m q^{b}-1 / 2-m}{q^{k}-1}\right\rfloor=\Delta m+m q^{b} \Theta$, we conclude $n \notin\left[\Delta m, \Delta m+m q^{b} \Theta\right]$ for $2 \leq m \leq q^{k-b}$.

It remains to show $n \notin\left[\Delta m, \Delta m+m q^{b} \Theta+1, \Delta(m+1)-1\right]=: I_{m}$ for all $2 \leq m \leq q^{k-b}-1$. If $n \in I_{m}$, then we can write $n=\Delta m+m q^{b} \Theta+x$ with $x \geq 1$ and $m q^{b} \Theta+x<\Delta$, so that $q^{k} \cdot\left(m q^{b} \Theta+x\right)=\Delta m+m q^{b} \Theta+$ $\left(x q^{k}-m q^{b}\right)<\Delta m+m q^{b} \Theta+x=n$, which contradicts Lemma 5

In other words, in the case of Theorem 1 (i), where $d_{2}=a d_{1}+b$ with $0<b<d_{1}$ and $a, b \in \mathbb{N}$, we have $u_{1} \geq q^{d_{2}-d_{1}} \cdot q^{d_{1}-b}+\frac{q^{(a+1) d_{1}}-1}{q^{d_{1}-1}}=\frac{q^{(a+2) d_{1}}-1}{q^{d_{1}-1}}$, which can be attained by an $d_{1}$-spread in $\mathbb{F}_{q}^{(a+2) d_{1}}$. Without the knowledge of $b$, we can state $u_{1} \geq q \cdot q^{d_{2}-d_{1}}+\left\lceil\frac{q^{d_{2}+1}-1}{q^{d_{1}}-1}\right\rceil$, which also improves Theorem 1 (i) and is tight whenever $d_{2}+1$ is divisible by $d_{1}$. Summarizing our findings we obtain:

Theorem 10. For a non-empty $q^{r}$-divisible set $\mathcal{N}$ of $k$-spaces in $\mathbb{F}_{q}^{v}$ the following bounds on $n=\# \mathcal{N}$ are tight.

(i) We have $n \geq q^{k}+1$ and if $r \geq k$ then either $k$ divides $r$ and $n \geq \frac{q^{k+r}-1}{q^{k}-1}$ or $n \geq \frac{q^{(a+2) k}-1}{q^{k}-1}$, where $r=a k+b$ with $0<b<k$ and $a, b \in \mathbb{N}$.

(ii) Let $q^{r}$ divide $n$. If $r<k$ then $n \geq q^{k+r}-q^{k}+q^{r}$ and $n \geq q^{k+r}$ otherwise.

While the smallest cardinality of a non-empty $q^{r}$-divisible set of $k$-spaces over $\mathbb{F}_{q}$ has been determined, the spectrum of possible cardinalities remains widely unknown. For $k=1$ [6, Theorem 12] states that either $n>r q^{r+1}$ or there exists integers $a, b$ with $n=a\left[\begin{array}{c}r+1 \\ 1\end{array}\right]_{q}+b q^{r+1}$ and bounds for the maximum excluded cardinality have been determined in [1]. However, Lemma 5] and Lemma 6, applied via Proposition 8, give restrictions going far beyond Theorem 10 . For $q=2, r=3, k=2$, and $n \leq 81$ we exemplarily state that only $n \in\{21,32,33,42$, $43,44,52, \ldots, 55,62, \ldots, 66,72, \ldots, 78\}$ might be attainable. The mentioned constructions cover the cases $n \in$ $\{21,32,42,53,63,64,74\} \subseteq\{21 a+32 b: a, b \in \mathbb{N}\}$. Replacing the lines by their contained 3 points, we obtain $2^{4}$ divisible sets of 1-spaces in $\mathbb{F}_{q}^{v}$ of cardinality $3 n$, for which two further exclusion criteria have been presented in [6], excluding the cases $n \in\{33,44\}$. [6, Lemma 23] is based on a cubic polynomial obtained from (1) and (2), similar to the quadratic polynomial from Lemma 6 obtained from (1). Here, the presence of $k$ additional $b_{i}$-variables may make the analysis more difficult for $k>1$. For a $q^{r}$-divisible set $\mathcal{N}$ of 1 -spaces we have that $\mathcal{N} \cap H$ is $q^{r-1}$-divisible for every hyperplane $H$, which allows a recursive application of the linear programming method. For $k>1$ we need to consider $k$-spaces and $k-1$-spaces in $H$, see [6, Section 6.3], which makes the bookkeeping more complicated.

\section{REFERENCES}

[1] D. Heinlein, T. Honold, M. Kiermaier, S. Kurz, and A. Wassermann, Projective divisible binary codes., The Tenth International Workshop on Coding and Cryptography 2017, 10 pages.

[2] J. Bierbrauer and Y. Edel, A family of 2-weight codes related to BCH-codes, Journal of Combinatorial Designs 5 (1997), no. 5, 391.

[3] A. Blokhuis, A.E. Brouwer, and H.A. Wilbrink, Heden's bound on maximal partial spreads, Discrete Mathematics 74 (1989), no. 3, $335-339$.

[4] P. Delsarte, An algebraic approach to the association schemes of coding theory, Philips research reports (1973), no. 10, 103.

[5] O. Heden, On the length of the tail of a vector space partition, Discrete Mathematics 309 (2009), no. 21, 6169-6180.

[6] T. Honold, M. Kiermaier, and S. Kurz, Partial spreads and vector space partitions, Network Coding and Subspace Designs (M. Greferath, M.O. Pavčević, N. Silberstein, and A. Vazquez-Castro, eds.), Springer, to appear.

[7] S. Kurz, Packing vector spaces into vector spaces, The Australasian Journal of Combinatorics 68 (2017), no. 1, 122-130.

Department of Mathematics, University of BAyreuth, 95440 BAyReuth, Germany

E-mail address: sascha.kurzeuni-bayreuth.de 